\title{
Characterization of Mechanical Properties of Acrylonitrile Butadiene Styrene (Abs) Parts Using Grey-Based Taguchi Method
}

\author{
Emmanuelle R. Biglete, Edward B.O. Ang \\ Mapúa University, Intramuros, Manila 1002, Philippines
}

\begin{abstract}
Additive manufacturing, sometimes called 3D printing, has been a very useful manufacturing process for the last decades. The mechanical properties of a part determine its suitable applications, operating conditions, and limitations. This study characterizes the mechanical properties of $A B S$ parts made by fused deposition modelling to achieve its optimum functionality. The build parameters like raster angle, build orientation, layer thickness, bead width, and infill play a vital role on the overall quality of the part especially its mechanical properties. Taguchi's parameter design was used to facilitate the number and method of sampling for the design of experiment. During testing, three tests were done for each parameter combination: compression, flexural, and impact tests. For data analysis, the signalto-noise ratio and ANOVA techniques of Taguchi method were used to determine the effect of the parameter variability on the mechanical responses of the material. This study presents information that relate the main effects of the printing conditions to the mechanical properties of ABS-printed parts referenced to ASTM dimensions and procedures. Grey relational analysis was also integrated with Taguchi method for optimizing the selected mechanical properties with multiple response characteristics which uses grey relational grade as the performance indicator. The experimental results revealed that unique sets of level combinations optimize the selected mechanical properties. Confirmation experiments were done to compare the predicted and experimental values. The results of the confirmation tests yielded an improvement in the grey relational grade which indicates a successful optimization of the process parameters.
\end{abstract}

Keywords: Design of experiment; fused deposition modelling; Grey relational analysis;

Optimization; Signal-to-noise ratio 